\title{
BMJ Open Impact of the communication and patient hand-off tool SBAR on patient safety: a systematic review
}

\author{
Martin Müller, ${ }^{1,2}$ Jonas Jürgens, ${ }^{2}$ Marcus Redaèlli, ${ }^{2}$ Karsten Klingberg, \\ Wolf E Hautz, ${ }^{1}$ Stephanie Stock ${ }^{2}$
}

To cite: Müller $M$, Jürgens $\mathrm{J}$, Redaèlli $\mathrm{M}$, et al. Impact of the communication and patient hand-off tool SBAR on patient safety: a systematic review. BMJ Open 2018;8:e022202. doi:10.1136/ bmjopen-2018-022202

- Prepublication history and additional material for this paper are available online. To view these files, please visit the journal online (http://dx.doi. org/10.1136/bmjopen-2018022202).

Received 17 February 2018

Revised 11 July 2018

Accepted 26 July 2018
Check for updates

(C) Author(s) (or their employer(s)) 2018. Re-use permitted under CC BY-NC. No commercial re-use. See rights and permissions. Published by BMJ.

${ }^{1}$ Department of Emergency Medicine, Inselspital, Bern University Hospital, University of Bern, Bern, Switzerland ${ }^{2}$ Institute of Health Economics and Clinical Epidemiology, University Hospital of Cologne, Cologne, Germany

Correspondence to Dr Martin Müller; martin.mueller2@insel.ch

\section{ABSTRACT}

Objectives Communication breakdown is one of the main causes of adverse events in clinical routine, particularly in handover situations. The communication tool SBAR (situation, background, assessment and recommendation) was developed to increase handover quality and is widely assumed to increase patient safety. The objective of this review is to summarise the impact of the implementation of SBAR on patient safety.

Design A systematic review of articles published on SBAR was performed in PUBMED, EMBASE, CINAHL, Cochrane Library and PsycINFO in January 2017. All original research articles on SBAR fulfilling the following eligibility criteria were included: (1) SBAR was implemented into clinical routine, (2) the investigation of SBAR was the primary objective and (3) at least one patient outcome was reported.

Setting A wide range of settings within primary and secondary care and nursing homes.

Participants A variety of heath professionals including nurses and physicians.

Primary and secondary outcome measures Aspects of patient safety (patient outcomes) defined as the occurrence or incidence of adverse events.

Results Eight studies with a before-after design and three controlled clinical trials performed in different clinical settings met the inclusion criteria. The objectives of the studies were to improve team communication, patient hand-offs and communication in telephone calls from nurses to physicians. The studies were heterogeneous with regard to study characteristics, especially patient outcomes. In total, 26 different patient outcomes were measured, of which eight were reported to be significantly improved. Eleven were described as improved but no further statistical tests were reported, and six outcomes did not change significantly. Only one study reported a descriptive reduction in patient outcomes.

Conclusions This review found moderate evidence for improved patient safety through SBAR implementation, especially when used to structure communication over the phone. However, there is a lack of high-quality research on this widely used communication tool.

Trial registration none

\section{INTRODUCTION}

Patient safety is crucial for the delivery of effective, high-quality healthcare ${ }^{1}$ and is

\section{Strengths and limitations of this study}

- This systematic review was conducted in accordance with the Cochrane Collaboration standards using a validated tool for quality assessment of the identified studies.

- Five well-known databases as well as the references of the included studies were searched using an open search strategy.

- Reliability of the study selection, data extraction and rating of the study quality was ensured using two independent reviewers.

- Studies in which SBAR (situation, background, assessment and recommendation) was part of a larger quality improvement initiative and outcomes that did not measure the incidence of adverse events were not included in this review.

- The heterogeneity of the studies impeded to test for publication bias or to perform a meta-analysis.

defined by the World Alliance for Patient Safety of WHO as 'the reduction of risk of unnecessary harm associated with healthcare to an acceptable minimum'. ${ }^{2}$ To illustrate the impact of patient safety on healthcare quality, the incidence of adverse events is commonly cited. Following the definition of Brennan $e t$ $a l l^{3}$ adverse events are injuries that are caused by medical conduct resulting in prolonged hospitalisation and/or disability at the time of discharge. The Joint Commission reported that poor communication is a contributing factor in more than $60 \%$ of all hospital adverse events they reviewed. ${ }^{4}$ Poor communication is found in many different healthcare settings and is especially prominent in patient hand-offs and settings where fast and effective management is indispensable. Such settings include the perioperative period, ${ }^{5}$ the intensive care unit (ICU) ${ }^{6}$ and the emergency department. ${ }^{7}$ The components and processes of communications are complex and prone to misunderstanding. ${ }^{8}$ To overcome these barriers, communication strategies are desirable, which take little time and effort to 


\begin{tabular}{|c|c|c|c|c|}
\hline & & Questions & Description & Example \\
\hline S & Situation & $\begin{array}{l}\text { What is going on } \\
\text { with the patient? } \\
\text { What is the situation } \\
\text { you are calling/ } \\
\text { communicate } \\
\text { about? }\end{array}$ & $\begin{array}{l}\text { First, the speaker presents the situation, by } \\
\text { identifying himself, stating the patient's name and } \\
\text { briefly describing the problem }\end{array}$ & $\begin{array}{l}\text { 'Dr Preston, I'm calling } \\
\text { about Mr Lakewood, who's } \\
\text { having trouble breathing' }\end{array}$ \\
\hline A & Assessment & $\begin{array}{l}\text { What is the } \\
\text { problem? }\end{array}$ & $\begin{array}{l}\text { Then specific information on vital signs, recent } \\
\text { laboratories and other quantitative or qualitative } \\
\text { data related to the patient's current state are } \\
\text { provided. This section can include a provisional } \\
\text { diagnosis or clinical impression }\end{array}$ & $\begin{array}{l}\text { 'I don't hear any breath } \\
\text { sounds in his right } \\
\text { chest. I think he has a } \\
\text { pneumothorax' }\end{array}$ \\
\hline $\mathrm{R}$ & Recommendation & $\begin{array}{l}\text { What is the } \\
\text { next step in the } \\
\text { management of the } \\
\text { patient? }\end{array}$ & $\begin{array}{l}\text { An informed suggestion for the continued care } \\
\text { of the patient has to be made by the speaker. } \\
\text { The immediate need is explained clearly and } \\
\text { specifically, including what is necessary to address } \\
\text { the problem }\end{array}$ & $\begin{array}{l}\text { 'I need you to see him right } \\
\text { now. I think he needs a } \\
\text { chest tube' }\end{array}$ \\
\hline
\end{tabular}

The tool is available for download from the website of the Institute for Healthcare Improvement. ${ }^{9}$

complete, deliver comprehensive information efficiently, encourage interprofessional collaboration and limit the probability of error. ${ }^{9-11}$ The SBAR (situation, background, assessment, recommendation) instrument (see table 1) and its derivatives ISBAR, SBAR-R, ISBARR and ISOBAR fulfil this need and are widely used in different healthcare facilities as a communication and hand-off tool both intraprofessionally and interprofessionaly. ${ }^{12-15}$ By virtue of a clear structure, SBAR calls for the provision of all relevant information, organised in a logical fashion. ${ }^{16}$ Furthermore, it enables a preparation before the communication process, ${ }^{16}{ }^{17}$ and because sender and receiver share the same mental model, understanding and awareness are expected to be higher. ${ }^{18}$ Besides, it reduces inhibitions especially in hierarchical context by encouraging the sender to provide a personal assessment and suggestion of the situation ('Recommendation'). ${ }^{19}$ The SBAR tool is regarded as a communication technique that increases patient safety and is current 'best practice' to deliver information in critical situations. ${ }^{1620}$

A number of studies have investigated 'soft' outcomes such as employee satisfaction ${ }^{21}{ }^{22}$ and interdisciplinary communication $^{19}{ }^{23}$ in relation to SBAR. Positive resonances of employees after the introduction of SBAR were reported ${ }^{24-28}$ with improvements of the communication perception and interdisciplinary teamwork ${ }^{29-33}$ as well as the quality of the communication. ${ }^{34-40}$ Especially in patient hand-off, the quality of the communication and the completeness of transferred information was increased after the implementation of SBAR ${ }^{41-44}$ Furthermore, less time was needed for the patient hand-off in several studies. ${ }^{40} 4245$
However, the actual effect of SBAR on patient outcome is unclear. The wide adoption of SBAR (or any other communication strategy) without proven benefit may paradoxically limit improvements because a problem presumably solved will be less addressed. Thus, the purpose of this systematic review is to summarise the available evidence for and evaluate the impact of the implementation of SBAR in clinical settings on patient safety as measured by the incidence of adverse events.

\section{METHODS}

\section{Search strategy}

A systematic search for articles published on SBAR was performed in PUBMED, EMBASE, CINAHL, Cochrane Library and PsycINFO via OvidSP. The search was conducted in January 2017. It was augmented by a review of the references of all articles included. Search terms used in all electronic medical databases were SBAR, ISBAR, SBAR-R, ISBARR and ISOBAR (combined as text words with the Boolean operator 'OR'). The detailed search strategy is provided in online supplementary appendix A. No restrictions were applied in terms of time, language or type of article. No review protocol exists.

\section{Eligibility criteria}

All original research articles on SBAR fulfilling the following eligibility criteria were included:

- SBAR was implemented into clinical routine,

- The investigation of SBAR was the primary objective of the study (as opposed to, for example, SBAR as part of a larger quality improvement initiative), 
- At least one patient outcome was reported (eg, mortality or secondary ICU admission). In accordance with the definition of $\mathrm{WHO},{ }^{2}$ aspects of patient safety (patient outcomes) were defined through outcome parameters measuring the occurrence or incidence of adverse events.

Exclusion criteria were:

- Articles that only describe the SBAR tool but provide no evaluation data on patient outcome,

- Studies that report a larger project in which SBAR was not the main intervention under investigation (because in such studies the attribution of any effect to SBAR is impossible),

- Studies that only report, survey outcomes or team perceptions.

\section{Selection of studies}

Studies were evaluated in two steps: (1) Two trained reviewers (JJ, MM) reviewed all abstracts and titles for eligibility. (2) If the eligibility of an article could not be clearly determined, the article was included for further full-text evaluation in a second step.

In case of dissent, the reviewers solved the divergence by consensus or, if necessary, by involving a third reviewer (MR).

\section{Data extraction}

The following data were extracted out of the included articles using a predefined form in Microsoft Excel for Mac 2011 (V.14.7.2; Microsoft, Redmond, Washington, USA): characteristics of the study (study setting, study design and information to evaluate the risk of bias; see below), characteristics of the study population and possible control group (type and number of trained people), characteristics of the intervention (type and duration) and outcome data on patients' safety including time/period of measurement). To ensure high accuracy and completeness of the data extraction by MM and JJ, data extraction was checked by KK.

\section{Quality assessment}

Methodological quality of the studies included was assessed with the 'Quality Assessment Tool for Quantitative Studies' developed by the Effective Public Health Practice Project Canada. ${ }^{46}$ The tool is recommended by the Cochrane Collaboration ${ }^{47}$ as it evaluates the full range of quantitative study designs. It has been evaluated for interrater reliability, content and construct validity. ${ }^{48}$ The identified studies were assessed on 18 criteria in six domains (selection bias, study design, confounders, blinding, data collection methods, as well as withdrawals and drop-outs). Studies were rated as 'strong', 'moderate' or 'weak' in each domain. An accompanying algorithm consolidates the six ratings into an overall score.

Two reviewers (JJ, MM) independently assessed the quality of each study. The final assessment of each study was determined by consensus between the two reviewers and, if necessary, by involving a third reviewer (WEH).

\section{Data synthesis}

The intraclass correlation coefficient (ICC) using Stata's ICC command with a two-way mixed-effects model was calculated to quantify the rater agreement on study inclusion as well as on quality ratings of the studies included.

The heterogeneity of reported study designs, outcome measures, settings and forms of SBAR interventions does not allow to pool data across the studies that met the inclusion criteria. Characteristics and results of the studies are presented in a narrative form.

\section{Patient and public involvement}

No patients were involved in the design, recruitment or conduct of the study. The results of this review will not be disseminated to patients included in the trials of the review.

\section{RESULTS}

\section{Systematic review process}

Article identification and inclusion is depicted in figure 1. The literature search identified 1053 articles. Seven hundred and one (701) articles remained after exclusion of duplicates; 607 articles were excluded after reviewing the titles and abstracts. Of the remaining 94 articles analysed in full text, 11 articles were included into this review. The rater agreement on inclusion was ICC 0.90 (95\% CI 0.86 to 0.94$)$. No additional studies were identified through screening of the references of the included articles.

\section{Quality assessment}

Rater agreement on the studies quality ratings was excellent (ICC $0.85,95 \% 0.78$ to 0.90 ).

The randomised controlled trial (RCT) by Field $e t a l^{49}$ was rated as 'strong' and one controlled trial by Randmaa $e t a l^{37}$ as 'moderate' in the overall study quality, while the remaining nine studies were rated as 'weak' (figure 2).

Three studies were rated as strong in the study design category as they were controlled clinical trials. ${ }^{37450}$ Eight studies used a before-after study design resulting in a weak rating in the study design category.

Except for the study by Christie and Robinson, ${ }^{41}$ in which the selected individuals were not described in sufficient detail, the study quality regarding selection bias was rated as 'moderate'.

The study by Field $e t a t^{49}$ used a RCT as a design with facility as a randomisation unit. Thus, by study design, the results were controlled for potential (known and unknown) confounders such as infrastructure, patient safety culture and management.

No other study controlled for confounders in the study design or analysis (weak rating).

While main outcomes, study objectives and the applied SBAR intervention were described in all studies, blinding was not described in any but one of the studies $(9.1 \%)$, resulting in a 'moderate' rating in this category. In one of the controlled trials, ${ }^{49}$ the reviewers who rated the patient 


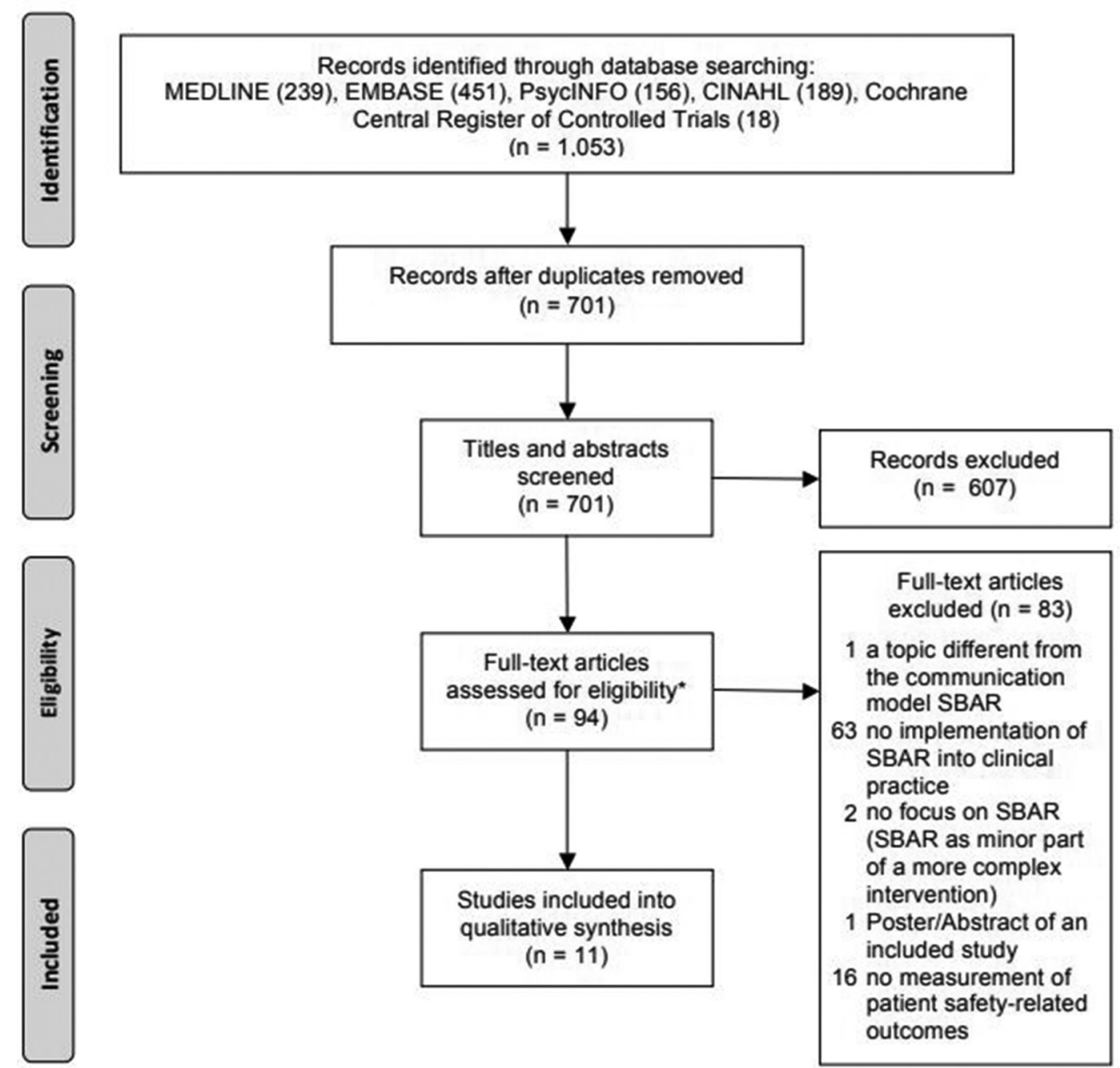

Figure 1 Flowchart of the systematic review process. SBAR, situation, background, assessment, recommendation. *No additional studies were identified through screening of the references of the included articles.

safety outcome were blinded in regard to the intervention (strong rating).

Overall, there was a lack of reporting on statistical tests ${ }^{51-54}$ and number of persons that were trained. ${ }^{41} 495153-55$ Sample size calculations to ensure sufficient power were not reported in any of the studies.

\section{Study setting and study characteristics}

Eight of the analysed studies $(72.7 \%)$ used a before-after intervention design, ${ }^{41}{ }^{51-57}$ while in two studies (18.2\%) a non-RCT ${ }^{37} 50$ and in one study $(9.2 \%)$ a RCT $^{49}$ were reported.

All identified articles were published in recent years (2006-2016). Eight $(72.7 \%)$ of the 11 studies were conducted in North America, ${ }^{49-545657}$ and the remaining three $(27.3 \%)$ were performed in Europe. ${ }^{374155}$

The studies focused on three different study sites: (1) hospitals in seven studies $(63.6 \%), 3741505153-55$ (2) a rehabilitation centre (geriatric/musculoskeletal unit) in one study ${ }^{52}(9.2 \%)$ and (3) nursing homes in three studies (27.3\%). ${ }^{45657}$ Four of the studies that introduced the SBAR tool into a hospital setting restricted the intervention to specific units (anaesthesiological, ${ }^{50}$ surgical $^{37}$ or medicosurgical ${ }^{5455}$ ) while three trials introduced the SBAR tool to all departments. ${ }^{415153}$ Nurses were trained in the use of SBAR in all studies. In five studies (45.5\%), additionally other clinical staff, for example, physicians, were trained also. ${ }^{374150-52}$ The number of staff members trained ranged from $38^{50}$ to $155^{37}$, but was not specified in five studies ${ }^{41} 49515355$ (online supplementary appendix B).

The study period was mainly dependent on the time period that the patient outcomes were measured and ranged between $2^{50}$ and 24 months ${ }^{3756}$ and was not specified in two studies. ${ }^{4153}$

\section{Intervention targets}

A detailed description of the wide range of implementation strategies of SBAR in the studies included is provided in online supplementary appendix C.

In two studies $(18.2 \%)$, the aim of the intervention was to improve team communication in general ${ }^{51}{ }^{52}$ while five studies $(45.5 \%)$ focused on patient hand-offs either between nurses or interprofessional. ${ }^{37415354}$ The four 


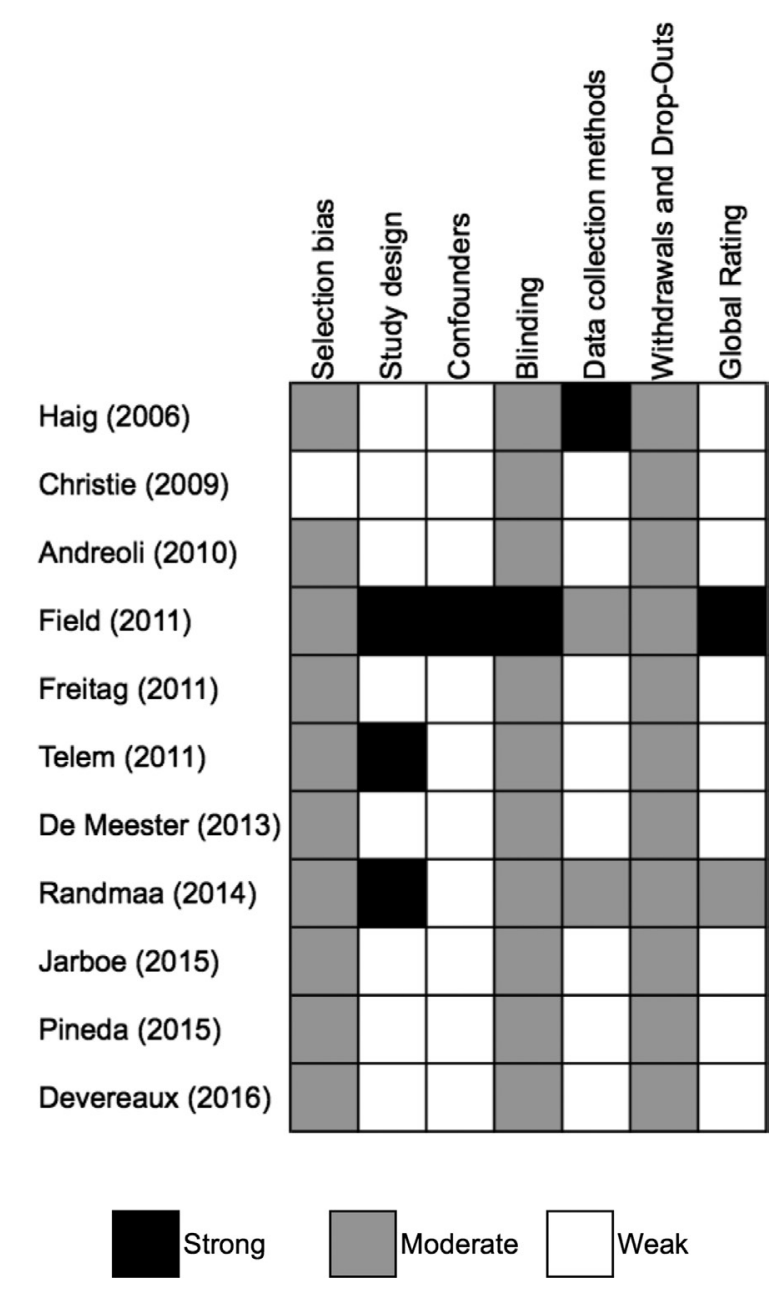

Figure 2 Quality assessment of the included studies.

remaining studies $(36.4 \%)$ aimed to improve communication in a particular situation such as telephone calls between nurses and physicians for anticoagulation management ${ }^{49}$ or in case of patient deterioration. ${ }^{55-57}$

Implementation strategies were educational programmes (seven studies ${ }^{449515254-56}$ ), organisational/human support (seven studies ${ }^{37} 414951-54$ ) and interactive teaching (seven studies $^{37} 415052535556$ ) including group discussions and role play. Additional SBAR trigger tools (poster, pocket cards, telephone stickers) were used in six studies $(54.5 \%){ }^{37414951-53}$

\section{Patient outcome}

All studies included assessed the effect of SBAR implementation on the outcome of inpatients, none the outcome of outpatients. The patient outcomes and outcome measurements varied widely over the identified studies (online supplementary appendix D). Three studies (27.3\%) measured general patient outcomes such as adverse patient/drug events, ${ }^{41} 5051$ while the remaining eight studies $(72.7 \%)$ used specific adverse event outcomes such as anticoagulation-related ${ }^{49}$ and patient fall-related adverse events ${ }^{52-54}$ as well as unplanned events such as ICU admissions, ${ }^{55}$ death/cardiac arrests ${ }^{41}$ and transfer to hospitals. ${ }^{56}{ }^{57}$ Other patient outcomes included methicillin-resistant Staphylococcus aureus (MRSA) bacteraemias $^{41}$ and catheter-associated urinary tract infection rates. ${ }^{53}$

The duration of measurement of the patient outcomes in the pre/controlled phase respectively the post/intervention phase ranged from 1 month $^{5054}$ to 12 months ${ }^{37}$ and was not reported in three studies. ${ }^{415253}$ Three of the studies $^{375155}$ controlled the use of SBAR by staff survey or review of medical records and identified high use rates within daily routine.

\section{Effect of SBAR on patient outcomes \\ Overall summary}

The main study characteristics and the effects of SBAR on the studied patient outcomes are summarised in table 2.

In total, 26 different patient outcomes were measured. Of these, eight outcomes measured in five studies $^{37} 49545557$ significantly improved and 11 patient outcomes measured in four before-after studies ${ }^{41}{ }^{51-53}$ are described as improving without the report of a statistical test. Six outcomes did not change significantly. One study descriptively reported an increase of adverse events, ${ }^{52}$ and none found a significant reduction of patient safety. The reported results of the studies are shown in detail in online supplementary appendix D.

\section{Team communication in general}

While one of the two before-after studies that focused on team communication in genera ${ }^{51}$ found a reduction of adverse patient as well as of drug events, a study that focused on falls in a rehabilitation centre ${ }^{52}$ found mixed results with a decrease in major falls, but an increase in the incidence of overall falls. Both studies did not provide a statistical analysis of their results.

\section{Patient hand-off}

All but one ef $^{50}$ of the five studies ${ }^{3741} 505354$ that focused on patient hand-offs reported an improvement of patient safety. Two before-after studies focused on patient hand-off between nursing shifts. ${ }^{53}{ }^{54}$ A reduction in the number of patient falls was reported in both studies. In addition, restrained patients rate and catheter-associated urinary tract infection rate decreased about one-third in one of these studies. ${ }^{53}$ Both studies that focused on patient hand-offs between physicians and nurses reported an improvement in patient safety-related outcome. ${ }^{37} 41$

In their controlled clinical trial, Randmaa et $a i^{37}$ reported that the critical incidence reporting system (CIRS) events due to communication breakdowns in the department of anaesthesiology of two clinics decreased significantly from $31 \%$ to $11 \%$. The before-after study performed in a hospital by Christie and Robinson ${ }^{41}$ found a reduction in hospital mortality $(-11 \%)$, MRSA bacteraemias $(-83 \%)$, adverse events $(-65 \%)$ and cardiac arrests $(-8 \%)$ after SBAR implementation (no further statistical analysis reported).

The controlled clinical by Telem et $a \tilde{\nu}^{0}$ evaluated the effect of SBAR versus no-SBAR training on patient 
Table 2 Study characteristics and outcomes sorted by effect on patient safety, study design and year

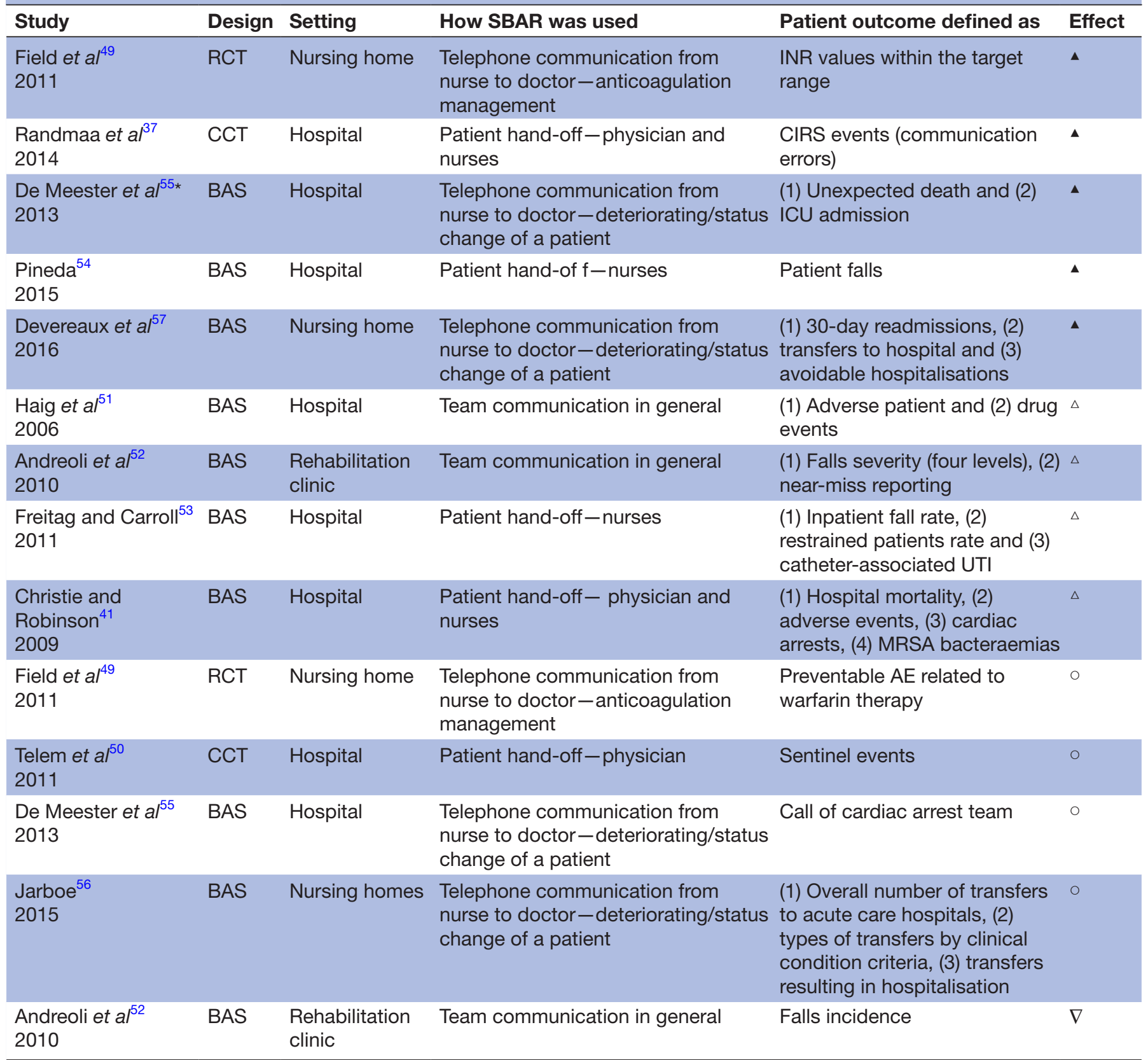

If a study reported outcomes with different effects on patient safety, the study results are listed separately.

$\Delta$, statistically significant evidence for improvement; $\Delta$, descriptive evidence for improvement (no statistical test reported); $\odot$, no significant evidence of a change; $\nabla$, descriptive reduction of patient safety.

${ }^{*}$ And nursing hand-off (between shifts).

AE, adverse event; BAS, before-after study; CCT, clinical controlled trial; CIRS, critical incident reporting system; ICU, intensive care unit; INR, international normalised ratio; MRSA, methicillin-resistant Staphylococcus aureus; RCT, randomised controlled trial; SBAR, situation, background, assessment and recommendation; UTI, urinary tract infection.

hand-offs by physicians on surgical wards. The number of identified sentinel events was not statistically different between the study groups. One sentinel event was reported over the whole study period.

Telephone communication between nurse and physician

Three trials tried to increase the quality of telephone communication between nurse and physician when nurses reported deterioration or other status changes of patients. ${ }^{55-57}$ Two studies reported significant improvements in the study patient outcome under investigation while the study of Devereaux $e t a \bar{l}^{57}$ could not find a significant change.

Field et at $t^{49}$ showed a statistically significant improvement in the management of anti-coagulated patients in nursing centres using a randomised controlled design: the international normalised ratio (INR) value of patients 
was $4.5 \%$ more time within the therapeutic range in the intervention homes than in control homes $(95 \%$ CI $3.1 \%$ to $8.7 \%$ ). They further reported a non-significant reduction of adverse warfarin-related events in the intervention homes (OR $0.9,95 \%$ CI 0.5 to 1.4 ).

De Meester et a $\iota^{\tilde{5} 5}$ (before-after study) reported that the number of unexpected death was significantly decreased from 0.99 to 0.34 per 1000 admissions $(p<0.001)$, while ICU admissions increased (13.1 to 14.8 per 1000 admissions) without a significant difference in the frequency with which a cardiac arrest team was called.

Devereaux et $a l^{57}$ studied transfers from nursing homes to acute care hospitals using a before-after trial and found a significant reduction in 30-day readmissions (0.12 vs $0.04, \mathrm{p}=0.012)$ and avoidable hospitalisations $(0.15$ vs $0.05, \mathrm{p}=0.007)$. Jarboe $^{56}$ used a similar setting, but a longer study period (20 months vs 6 months) and could not find significant differences with regard to preventable patient transfers $(\mathrm{p}=0.927)$ or emergent patient transfer $(\mathrm{p}=0.565)$.

\section{DISCUSSION}

\section{Summary of main results}

The present systematic review assesses the effect of the implementation of the widely adopted communication strategy SBAR on patient-related outcomes. Because communication breakdowns have been repeatedly identified as a major source of adverse events and medical error, ${ }^{48} 59$ implementation of a strategy such as SBAR seems a valid remediation approach.

Eleven studies, eight with a before-after design and three controlled trials, met the inclusion criteria. SBAR was implemented through different strategies in three different clinical settings (hospitals, rehabilitation centre and nursing homes) and with a broad range of objectives to improve (1) team communication in general, (2) intradisciplinary and interdisciplinary patient hand-offs, and (3) communication in telephone calls from nurses to physicians. In total, 26 different patient outcomes were measured. Eight significantly improved, 11 were described as improving (but no further statistical test were reported), six outcomes did not change significantly and one study reported a descriptive reduction in patient outcomes. Study outcomes with statistical evidence for improvement included INR values within the target range ${ }^{49}$ and unplanned transfers to hospitals ${ }^{57}$ in nursing homes, as well as CIRS events due to communication errors,${ }^{37}$ patient falls, ${ }^{54}$ unexpected death and ICU admis$\operatorname{sions}^{55}$ in hospitals. The overall study quality was high or moderate in two studies only; all other studies showed a weak study quality.

\section{Quality of the evidence}

The strongest evidence identified in our review comes from a single RCT investigating the effect of SBAR implementation in nursing homes on anticoagulation management of patients under warfarin. ${ }^{27}$ However, because warfarin is increasingly substituted by direct oral anticoagulants less difficult to dose ${ }^{60}$ the relevance of this finding may cease over time. Furthermore, adverse events related to warfarin therapy, the primary outcome parameter in this study, did not differ significantly between the intervention and control group. We found further evidence that the use of SBAR in telephone communication to inform the physician of a deteriorating patient leads to (1) a significant decrease in unexpected death ${ }^{22}$ and (2) a significant reduction in transfers to hospitals, 30-day readmissions and avoidable hospitalisations from nursing homes. ${ }^{21}$ Therefore, SBAR implementation in telephone communication seems to positively affect patient outcome. However, one study conducted in a similar setting ${ }^{56}$ (nursing home, unplanned hospital admission) but with a longer study period could not find any significant difference between the preimplementation and postimplementation phase in the patient outcomes. One explanation for the differences in the findings might be that the use of SBAR (not reported in the two studies) decreased over time, thus the effect vanished.

Study periods were short at least in two trials ${ }^{50} 54$ (2 months only). As a consequence, only one sentinel event in one controlled clinical trial ${ }^{50}$ over the study period was reported.

Power calculations were missing in all studies. Thus, the lack of significant differences between the groups in these studies could not be interpreted adequately. Furthermore, in almost half of the reported outcomes, no statistical tests were performed. Notably, no study in our review found a significant increase in the occurrence of adverse events after to the implementation of SBAR, but Andreoli $e t a \tilde{l}^{2}$ descriptively reported an increase in fall incidence while the fall severity was reduced at the same time. This study's findings illustrate the difficulty with most of the studies findings included in the review. Some might argue that the implementation of SBAR in patient fall reporting has just led to an increased awareness regarding patient falls. Consequently, the reporting of patient falls and especially of less severe falls increased, resulting in a decrease of the patient fall severity overall.

It has been previously argued that downstream targets of educational interventions (such as the implementation of a specific communication strategy) are often difficult to assess due to possible dilution of the effect of any intervention. ${ }^{61}{ }^{62}$ Indeed, implementation of SBAR may only directly affect communication among health professionals, which in turn may or may not affect healthcare conduct, which then may result in altered patient outcome. Arguably, there are many other effective agents along this path that may dilute the effect of SBAR implementation on patient outcome. We would argue that because it has been possible in the past to relate adverse events to communication breakdowns, ${ }^{758} 59$ it should just as well be possible to demonstrate the effect on patient safety of interventions targeted at remediating such communication breakdowns. 
One reason for the current failure to demonstrate such effects may be that studies investigating the effect of SBAR on patient outcome are mostly of limited quality and yield heterogeneous results. Many studies identified were before-after studies. It is thus difficult to differentiate between changes attributed to the implementation of SBAR and changes attributable to other factors that had changed over time, such as increased awareness. Process measures in regard to parameters of communication were not measured in any of the included studies, but several not included studies suggest an improvement of communication through the implementation of SBAR. ${ }^{34-40}$ The lack of process measures within the included studies reduces internal validity and impedes the interpretation of the present results with regard to causation. Consequently, the unreflected adoption of SBAR may paradoxically limit improvements in healthcare communication because once a problem appears to be solved, less research will be conducted on it.

\section{Limitations}

This systematic review has some limitations. Efforts were undertaken to identify all relevant trials to evaluate the impact of SBAR implementation in clinical practice on patient safety. Five well-known databases as well as the references of the studies that met the inclusion criteria were searched using an open search strategy. No grey literature was searched, thus trials could have been missed. Further, we did not contact any author to ask for raw data to perform additional statistical analysis. Publication bias could not be assessed leading to an important source of bias. The heterogeneity of the data impeded a meta-analysis. This systematic review was conducted in accordance with the Cochrane Collaboration standards using a validated tool for quality assessment of the identified studies. Reliability of the study selection, data extraction and rating of the study quality was ensured using two independent reviewers. We did not differentiate the broad range of adverse events or sentinel events, but subsume them under patient safety/outcome in order to provide a first insight into the relationship between SBAR and patient safety. The inclusion criteria were restricted to trials that reported at least one 'hard' patient outcome parameter to evaluate SBAR's impact on patient safety. Evidence of improvement of potentially 'soft' outcomes such as an increase in employee satisfaction ${ }^{21} 22$ and interdisciplinary communication $^{193}$ with improvements of the communication perception, interdisciplinary teamwork, ${ }^{29-33}$ completeness ${ }^{41-44}$ and efficiency ${ }^{40} 4245$ of the communication were not reported in this review. Last, trials in which SBAR was a minor component of a complex intervention only were not included in this review. These trials may contain potential evidence for an improvement of patient safety through the implementation of SBAR.

\section{Implications for practice and research}

Five of the studies ${ }^{379} 44557$ including the two moderate/ high-quality studies found significantly improved patient safety outcomes. Four other before-after studies ${ }^{41}$ 51-53 reported descriptive improved patient outcomes. On the one hand, these findings emphasise the potential importance of implementation of SBAR in the clinical practice to improve (1) telephone communication from nurse to doctors in critical situations, (2) general patient hand-off as well as (3) team communication in general. However, the quality of the evidence is low and four studies ${ }^{495} 5556$ reported no significant changes of other relevant outcomes and even a descriptive increase of patient falls also. ${ }^{52}$ Best evidence was found in telephone communication between nurses and physicians. This should raise awareness and demands future highquality research as the unreflected adoption of SBAR may paradoxically limit improvements in healthcare communication because once a problem appears to be solved, less research will be conducted on it.

\section{CONCLUSION}

In summary, many authors claim that SBAR improves patient safety. There is some evidence of the effectiveness of SBAR implementation on patient outcome, but this evidence is limited to specific circumstances such as communication over the phone. Especially high-quality studies are lacking. Future studies are needed to further demonstrate the benefit of SBAR in terms of patient safety and keep raising the awareness of communication errors. SBAR might be an adaptive tool that is suitable for many healthcare settings, in particular when clear and effective interpersonal communication is required.

Acknowledgements The authors want to thank the Gottfried und Julia BangerterRhyner-Foundation for their ad personam grant Young Talents in Clinical Research for MM.

Contributors All authors contributed to the conception of the review, analysis and interpretation of the results and the final approval of the manuscript. Study design: MM, JJ, MR, WEH, SS. Literature search and assessment (acquisition of data):

MM, JJ, KK. Drafting the manuscript: MM, JJ, MR, WEH. Critical revision of the manuscript for intellectual content: MM, JJ, MR, KK, WEH, SS.

Funding The authors have not declared a specific grant for this research from any funding agency in the public, commercial or not-for-profit sectors.

Competing interests WEH has received payment from the AO Foundation Zürich for educational consultations and congress invitations from Mundipharma Basel.

Patient consent Not required.

Provenance and peer review Not commissioned; externally peer reviewed.

Data sharing statement There are no additional data available.

Open access This is an open access article distributed in accordance with the Creative Commons Attribution Non Commercial (CC BY-NC 4.0) license, which permits others to distribute, remix, adapt, build upon this work non-commercially, and license their derivative works on different terms, provided the original work is properly cited, appropriate credit is given, any changes made indicated, and the use is non-commercial. See: http://creativecommons.org/licenses/by-nc/4.0/.

\section{REFERENCES}

1. Aspden P, Corrigan JM, Wolcott J, et al. Patient safety: achieving a new standard for care. Washington, DC: National Academies Press (US), 2004.

2. World Health Organisation. Conceptual Framework for the International Classification for Patient Safety. 2009 http://www.who. 
int/patientsafety/taxonomy/icps_full_report.pdf (accessed 10 Aug 2017).

3. Brennan TA, Leape LL, Laird NM, et al. Incidence of adverse events and negligence in hospitalized patients. Results of the Harvard Medical Practice Study I. N Engl J Med 1991;324:370-6.

4. The Joint Commission. Sentinel event data: root causes by event type 2004-2014. 2014 http://www.tsigconsulting.com/tolcam/wpcontent/uploads/2015/04/TJC-Sentinel-Event-Root_Causes_by_ Event Type 2004-2014.pdf (accessed 10 Aug 2017).

5. Haller G, Laroche T, Clergue F. Évènements indésirables et problèmes de communication en périopératoire. Annales Françaises d'Anesthésie et de Réanimation 2011;30:923-9.

6. Reader TW, Flin R, Mearns K, et al. Interdisciplinary communication in the intensive care unit. Br J Anaesth 2007;98:347-52.

7. Burley D. Better communication in the emergency department. Emerg Nurse 2011;19:32-6.

8. Dayton E, Henriksen K. Communication failure: basic components, contributing factors, and the call for structure. Jt Comm J Qual Patient Saf 2007;33:34-47.

9. Institute for Healthcare Improvement. SBAR technique for communication: a situational briefing model. http://www.ihi.org/ resources/Pages/Tools/SBARTechniqueforCommunicationASituat ionalBriefingModel.aspx (accessed 9 Aug 2017).

10. Nadzam DM. Nurses' role in communication and patient safety. $J$ Nurs Care Qual 2009;24:184-8.

11. National Patient Safety Agency. Recognising and responding appropriately to early signs of deterioration in hospitalised patients. London: National Patient Safety Agency, 2007.

12. von Dossow V, Zwissler B. Recommendations of the German Association of Anesthesiology and Intensive Care Medicine (DGAI) on structured patient handover in the perioperative setting: the SBAR concept. Anaesthesist 2016;65(Suppl 1):1-4.

13. Santhanakrishnan M, Gash A, Hopper S, et al. Improving quality of referral to consultation liaison service using SBAR communication tool to provide rapid and timely interventions to elderly patients in general hospital. Eur Geriatr Med 2013;4:S170.

14. Lee SY, Dong L, Lim YH, et al. SBAR: towards a common interprofessional team-based communication tool. Med Educ 2016;50:1167-8.

15. Riesenberg LA, Leitzsch J, Little BW. Systematic review of handoff mnemonics literature. Am J Med Qual 2009;24:196-204.

16. Dunsford J. Structured communication: improving patient safety with SBAR. Nurs Womens Health 2009;13:384-90.

17. Guise JM, Lowe NK. Do you speak SBAR? J Obstet Gynecol Neonatal Nurs 2006;35:313-4.

18. Powell SK. SBAR-it's not just another communication tool. Prof Case Manag 2007;12:195-6.

19. Donahue M, Miller M, Smith L, et al. A leadership initiative to improve communication and enhance safety. Am J Med Qual 2011;26:206-11.

20. NHS Institute for Innovation Improvement. The handbook of quality and service improvement tools: NHS Institute for Innovation and Improvement Coventry, 2010:247-51.

21. Landau S, Wellman LG. Small changes can streamline the handoff process in a staff-driven process improvement project. $J$ Obstet Gynecol Neonatal Nurs 2014;43(Suppl 1):S49.

22. Wathen B, Roth J, Dobyns E, et al. 681. Crit Care Med 2013;41(12 Suppl 1):A167.

23. Farley $\mathrm{H}$, Choy $\mathrm{H}$, Ellicott $\mathrm{A}$, et al. 168: utilization of the situationbackground-assessment-request, companion phones, and cell phones improves communication with consultants in the emergency department. Ann Emerg Med 2009;54:S52.

24. Manias T, Tomlinson J. Implementation and evaluation of the sbar tool in the communication between medical staff in obstetrics. Arch Dis Child Fetal Neonatal Ed 2011;96:Fa131.

25. McCrory $\mathrm{M}$, Aboumatar $\mathrm{H}$, Hunt $\mathrm{E}$. Communication during pediatric rapid response events: a survey of healthcare providers. Crit Care Med 2011;39:176.

26. Raymond M, Harrison MC. The structured communication tool SBAR (Situation, Background, Assessment and Recommendation) improves communication in neonatology. S Afr Med J 2014;104:850-2.

27. Renz SM, Boltz MP, Wagner LM, et al. Examining the feasibility and utility of an SBAR protocol in long-term care. Geriatr Nurs 2013;34:295-301.

28. Renz SM, Boltz MP, Capezuti E, et al. Implementing an SBAR communication protocol: a quality improvement project. Ann Longterm Care 2015;23:27-31.

29. Velji K, Baker GR, Fancott C, et al. Effectiveness of an adapted SBAR communication tool for a rehabilitation setting. Healthc Q 2008;11(3 Spec No.):72-9.
30. Gerard JC. The effect of a communication protocol implementation on nurse/physician collaboration and communication. Louisville: University of Louisville, 2011.

31. Edwards C, Woodard EK. SBAR for maternal transports: going the extra mile. Nurs Womens Health 2008;12:515-20.

32. Beckett CD, Kipnis G. Collaborative communication: integrating SBAR to improve quality/patient safety outcomes. J Healthc Qual 2009;31:19-28.

33. Albert B, Messina C, Parker M, et al. 759. Crit Care Med 2012;40(12 Suppl 1):1-328.

34. García-Sánchez MJ, Fernández-Guerrero C, López-Toribio P, et al. [Quality of the anesthesiologist written record during the transfer of postoperative patients: influence of implementing a structured communication tool]. Rev Esp Anestesiol Reanim 2014:61:6-14.

35. Mitchell C, Johnston D. Fast bleep audit-to determine the appropriateness of fast bleeps received and the quality of communication relayed. Anaesthesia 2014;69:47.

36. Panesar RS, Albert B, Messina C, et al. The effect of an electronic SBAR communication tool on documentation of acute events in the pediatric intensive care unit. Am J Med Qual 2016;31:64-8.

37. Randmaa M, Mårtensson G, Leo Swenne C, et al. SBAR improves communication and safety climate and decreases incident reports due to communication errors in an anaesthetic clinic: a prospective intervention study. BMJ Open 2014;4:e004268.

38. Woodhall LJ, Vertacnik L, McLaughlin M. Implementation of the SBAR communication technique in a tertiary center. J Emerg Nurs 2008;34:314-7.

39. Wyckoff A, Larsen K, Alexander R, et al. Huntsman cancer hospital sbar project. Oncol Nurs Forum 2009;36:12.

40. Zhu H, McCrea N, Kelsall W. G188 Improving the paediatric handover: quality, safety and SBAR. Arch Dis Child 2014;99:A82.

41. Christie $\mathrm{P}$, Robinson $\mathrm{H}$. Using a communication framework at handover to boost patient outcomes. Nurs Times 2009;105:13-15.

42. Cornell P, Gervis MT, Yates L, et al. Impact of SBAR on nurse shift reports and staff rounding. Medsurg Nurs 2014;23:334-42.

43. Moseley BD, Smith JH, Diaz-Medina GE, et al. Standardized signout improves completeness and perceived accuracy of inpatient neurology handoffs. Neurology 2012;79:1060-4.

44. Thompson JE, Collett LW, Langbart MJ, et al. Using the ISBAR handover tool in junior medical officer handover: a study in an Australian tertiary hospital. Postgrad Med J 2011;87:340-4

45. Sohi D, Scotney E, Sowerbutts $\mathrm{H}$, et al. Significantly improving the efficiency of communication in paediatrics. Arch Dis Child 2011;96:A90-A91.

46. National Collaborating Centre for Methods and Tools. Quality assessment tool for quantitative studies McMaster University, Hamilton, Canada 2008. 2010 http://www.nccmt.ca/resources/ search/14 (accessed 10 May 2018).

47. Higgins J, Green S, eds. Cochrane handbook for systematic reviews of interventions 4.2.6. 4 edn. Chichester, UK: John Wiley \& Sons, Ltd, 2006.

48. Armijo-Olivo S, Stiles CR, Hagen NA, et al. Assessment of study quality for systematic reviews: a comparison of the cochrane collaboration risk of bias tool and the effective public health practice project quality assessment tool: methodological research. J Eval Clin Pract 2012;18:12-18.

49. Field TS, Tjia J, Mazor KM, et al. Randomized trial of a warfarin communication protocol for nursing homes: an SBAR-based approach. Am J Med 2011;124:179.e1-7.

50. Telem DA, Buch KE, Ellis S, et al. Integration of a formalized handoff system into the surgical curriculum: resident perspectives and early results. Arch Surg 2011;146:89-93.

51. Haig KM, Sutton S, Whittington J. National Patient Safety Goals. SBAR: a shared mental model for improving communication between clinicians. Jt Comm J Qual Improv 2006;32:167-75.

52. Andreoli A, Fancott C, Velji K, et al. Using SBAR to communicate falls risk and management in inter-professional rehabilitation teams. Healthc Q 2010;13(Spec No):94-101.

53. Freitag M, Carroll VS. Handoff communication: using failure modes and effects analysis to improve the transition in care process. Qual Manag Health Care 2011;20:103-9.

54. Pineda RO. Improving patient outcomes and nurse satisfaction through nurse-to-nurse communication. Chester: Widener University, 2015.

55. De Meester K, Verspuy M, Monsieurs KG, et al. SBAR improves nurse-physician communication and reduces unexpected death: a pre and post intervention study. Resuscitation 2013;84:1192-6.

56. Jarboe DE. The effect of evaluating a quality improvement initiative on reducing hospital transfers of nursing home residents. Minneapolis: Walden University, 2015. 
57. Devereaux T, Devereaux T, Marchetti G, et al. Condition-specific sbar effect on transfers, hospitalizations, and 30 -day readmissions from long-term care to acute-care. J Am Med Dir Assoc 2016;17:B25.

58. Donaldson MS, Corrigan JM, Kohn LT. To err is human: building a safer health system. Washington, DC: National Academies Press, 2000.

59. Committee on Diagnostic Error in Health Care. Improving diagnosis in health care. Washington, DC: National Academies Press, 2015.

60. Sauter TC, Amylidi AL, Ricklin ME, et al. Direct new oral anticoagulants in the emergency department: experience in everyday clinical practice at a Swiss university hospital. Eur $J$ Intern Med 2016;29:e13-15.

61. Cook D, West C. Perspective: Reconsidering the focus on "outcomes research" in medical education: a cautionary note. Acad Med J Assoc Am Med Coll 2013;88:162-7.

62. Hautz WE, Kämmer JE, Exadaktylos A, et al. How thinking about groups is different from groupthink. Med Educ 2017;51:229.

63. Leonard M, Graham S, Bonacum D. The human factor: the critical importance of effective teamwork and communication in providing safe care. Qual Saf Health Care 2004;13(Suppl 1):i85-i90.

64. Powell SM, Kimberly Hill R. My copilot is a nurse-using crew resource management in the OR. Aorn J 2006;83:178-202. 